Ibn Al-Haitham Jour. for Pure \& Appl. Sci. 34 (2) 2021

Ibn Al Haitham Journal for Pure and Applied Science

Journal homepage: http://jih.uobaghdad.edu.iq/index.php/j/index

\title{
Parametric Models in Survival Analysis for Lung Cancer Patients
}

\author{
Layla A. Ahmed \\ layla.aziz@garmian.edu.krd \\ Department of Mathematics, College of Education, University of Garmian, Kurdistan Region, Iraq
}

Article history: Received, 7,January,2020, Accepted 20 ,Februry,2020, Published in April 2021

Doi: 10.30526/34.2.2617

\begin{abstract}
The aim of this study is to estimate the survival function for the data of lung cancer patients, using parametric methods (Weibull, Gumbel, exponential and log-logistic).

Comparisons between the proposed estimation method have been performed using statistical indicator Akaike information Criterion, Akaike information criterion corrected and Bayesian information Criterion, concluding that the survival function for the lung cancer by using Gumbel distribution model is the best. The expected values of the survival function of all estimation methods that are proposed in this study have been decreasing gradually with increasing failure times for lung cancer patients, which means that there is an opposite relationship failure times and survival function.
\end{abstract}

Keywords: Survival analysis, Weibull distribution, Gumbel distribution, Exponential distribution, Log-logistic distribution.

\section{Introduction}

Survival analysis is a branch of statistics which deals with the analysis of time to events, such as death in biological organisms and failure in mechanical systems. The topic of survival analysis is called reliability theory or reliability analysis in engineering, and duration analysis or duration modeling in economics or event history analysis in sociology [1]. In many applied sciences such as medicine, engineering and finance, amongst others, modeling and analyzing lifetime data are crucial.

Several lifetime distributions have been used to model such type of data. The quality of the executions and in a statistical analysis depends heavily on the presupposed probability distributions [2].

Parametric methods which involve the exponential, Weibull, lognormal, gamma and extreme value distribution have been widely used for fitting survival data [3]. 
Gumbel showed that the Weibull distribution and the type III smallest extreme value distribution are the same [4].

Log- logistic distribution is a very important reliability model as it fits well in many applied situations of reliability data analysis. Another advantage with the log- logistic distribution lies in its closed form expression for survival and failure rate functions that makes it important over log- normal distribution [5].

Salman and Farhan [6] estimated the survival function for the patients of lung cancer; they used several nonparametric estimation methods, and concluded that the shrinkage was the best method.

The main objective of this research is to estimate the survival function for the data of lung cancer patients, by using parametric methods and determine the best and most efficient distribution.

\section{Theoretical Part}

\subsection{Survival Analysis}

Survival function is the probability that a system or component will survive without failure during a specified time interval $[0, t]$ under given operating conditionals, denoted by $S$, which is defined as [7]:

$$
S(t)=P(T>t)=\int_{t}^{\infty} f(u) d u, t \geq 0
$$

Where, $T$ is a random variable, $t$ is the time of death.

The survival function $S(t)$ is the probability that the patient will survival till time $t$. Survival probability is usually assumed to approach zero as age increases, with:

$S(0)=1$

$\lim _{t \rightarrow \infty} S(t)=0$

$S(t)$ is decreasing and continuous from right side. It is linked with the failure distribution function $F(t)$ and in fact, it is the complement of it, i.e.:

$$
\begin{aligned}
& S(t)=1-F(t) \\
& \frac{d}{d t} S(t)=-f(t)
\end{aligned}
$$

\subsection{Life Time Distribution Function}

The life time distribution function, is defined as the complement of the survival function [1],

$F(t)=P(T \leq t)=1-S(t)$

If $F(t)$ is differentiable then the derivative, which is the density function of the lifetime distribution is,

$$
f(t)=\frac{d}{d t} F(t)
$$


The function $f(t)$ is sometimes called the event density; it is the rate of death or failure events per unit time.

\subsection{Hazard Function}

Hazard function is also known as the immediate failure rate [8]. This is the limit of the conditional probability that an item will fail in the time interval $[t, t+\Delta t]$ when we know that the item is functioning at time $t$ is

$$
\begin{aligned}
P(t<T \leq t+\Delta t / T>t) & =\frac{P(t<T \leq t+\Delta t)}{P(T>t)} \\
& =\frac{F(t+\Delta t)-F(t))}{S(t)}
\end{aligned}
$$

By dividing this probability by the length of the time interval, $\Delta t$, and letting $\Delta t \rightarrow 0$, we get the rate function $(h(t))$, and it is defined as:

$$
\begin{aligned}
& h(t)=\lim _{\Delta t \rightarrow 0} \frac{F(t+\Delta t)-F(t)}{S(t)} \\
& h(t)=\frac{d F(t)}{d t} \frac{1}{S(t)} \\
& h(t)=\frac{f(t)}{S(t)}
\end{aligned}
$$

Where $f(t)$ is the failure density functions, and $S(t)$ is the survival function.

Then,

$$
\begin{aligned}
& H(t)=\frac{-d \ln S(t)}{d t} \\
& S(t)=\exp \left(-\int_{0}^{t} h(s) d s\right)
\end{aligned}
$$

So hazard is instantaneous mortality rate conditional on previous survival, and the integrated form of cumulative hazard

$$
H(t)=\int_{0}^{t} h(s) d s=-\ln S(t)
$$

\section{Parametric Methods}

\subsection{Weibull Distribution}

The Weibull distribution is continuous distribution. It is one of the most widely applied life distributions in reliability analysis [9] and [10].

The probability density function is:

$$
f(t)=\left\{\begin{array}{cc}
\frac{\alpha}{\lambda}\left(\frac{t}{\lambda}\right)^{\alpha-1} e^{-\left(\frac{t}{\lambda}\right)^{\alpha}} & t>0 \\
0 & \text { elsewhere }
\end{array}\right.
$$


Where $(\alpha>0)$ is shape parameter and $(\lambda>0)$ is the scale parameter of the distribution. The mean and variance of Weibull distribution are respectively:

$$
\begin{aligned}
& E(t)=\lambda \Gamma\left(1+\frac{1}{\alpha}\right) \\
& V(t)=\lambda^{2}\left[\Gamma\left(1+\frac{1}{\alpha}\right)-\Gamma^{2}\left(1+\frac{1}{\alpha}\right)\right.
\end{aligned}
$$

The cumulative distribution function is defined as:

$$
F(t)=1-e^{-\left(\frac{t}{\lambda}\right)^{\alpha}}, t>0
$$

The survival function is defined as:

$$
S(t)=e^{-\left(\frac{t}{\lambda}\right)^{\alpha}}
$$

The failure rate (or hazard rate) function is given by:

$$
h(t)=\frac{\alpha}{\lambda}\left(\frac{t}{\lambda}\right)^{\alpha-1}=\alpha \lambda^{-\alpha} t^{\alpha-1}
$$

\subsection{Gumbel Distribution}

Gumbel (1958) denotes this distribution of the type I distribution of the smallest extreme, called the Gumbel distribution of the smallest extreme [8]. The Gumbel distribution is a very common distribution due to its global applicability in several fields and its wide applications [11] and [13]. The probability density function is:

$$
f(t)=\left\{\begin{array}{cc}
\frac{1}{\beta} \exp [-(z+\exp (-z))] & -\infty<t<\infty \\
0 & \text { elsewhere }
\end{array}\right.
$$

where $z=\frac{t-\mu}{\beta}, \mu$ is the location parameter, and $\beta$ is the scale parameter of the distribution.

The mean and variance of Weibull distribution are respectively:

$E(t)=\mu+\beta \gamma$,

$\gamma$ is Euler's constant $(0.577215)$

$$
V(t)=(\beta \pi)^{2} / 6
$$

The cumulative distribution function is defined as:

$$
F(t)=1-\exp [-\exp (-z)],-\infty<t<\infty
$$

The survival function is defined as:

$$
S(t)=\exp [-\exp (-z)]
$$

The failure rate (or hazard rate) function is given by:

$$
h(t)=\frac{\frac{1}{\beta} \exp [-(z+\exp (-z))]}{\exp [-\exp (-z)]}
$$




\subsection{Exponential Distribution}

The exponential distribution is a special case of two- parameter Weibull distribution (or gamma distribution) when the shape parameter is $(\alpha=1)$ in equation 10 , then the probability density function is [11]:

$$
f(t)=\left\{\begin{array}{cc}
\frac{1}{\lambda} e^{-\frac{t}{\lambda}} & t>0 \\
0 & \text { elsewhere }
\end{array}\right.
$$

where $\lambda>0$ is the scale parameter of the distribution, and the mean and variance of exponential distribution are respectively:

$$
\begin{aligned}
& E(t)=\lambda \\
& V(t)=\lambda^{2}
\end{aligned}
$$

The cumulative distribution function is defined as:

$$
F(t)=1-e^{-\frac{t}{\lambda}}, t>0
$$

The survival function is defined as:

$$
S(t)=e^{-\frac{t}{\lambda}}
$$

The failure rate (or hazard rate) is given by:

$$
h(t)=\frac{1}{\lambda}
$$

\subsection{Log- Logistic Distribution}

Log- logistic distribution is widely used in survival analysis when the failure rate function presents an unmoral shape [5]:

$f(t)=\left\{\begin{array}{cc}\frac{\beta}{\alpha}\left(\frac{t}{\alpha}\right)^{\beta-1}\left[1+\left(\frac{t}{\alpha}\right)^{\beta}\right]^{-2} & t>0 \\ 0 & \text { elsewhere }\end{array}\right.$

where $(\alpha>0)$ is scale parameter and $(\beta>0)$ is the shape parameter of the distribution.

The mean and variance are respectively [12],

$E(t)=\frac{\pi \alpha \beta^{-1}}{\sin \left(\pi \beta^{-1}\right)} \quad, \beta>0$

And

$V(t)=\frac{2 \pi \alpha^{2} \beta^{-1}}{\sin \left(2 \pi \beta^{-1}\right)}-\left[\frac{\pi \alpha \beta^{-1}}{\sin \left(\pi \beta^{-1}\right)}\right]^{2}, \beta>2$

The cumulative distribution function is defined as:

$$
F(t)=1-\left[1+\left(\frac{t}{\alpha}\right)^{\beta}\right]^{-1}
$$

Also the survival function is defined as: 


$$
S(t)=\left[1+\left(\frac{t}{\alpha}\right)^{\beta}\right]^{-1}
$$

The hazard function is given by:

$h(t)=\frac{\beta}{\alpha}\left(\frac{t}{\alpha}\right)^{\beta-1}\left[1+\left(\frac{t}{\alpha}\right)^{\beta}\right]^{-1}$

\section{Goodness of Fit Test}

In order to compare the distributions, we consider some other criterion like Akaike information Criterion (AIC), Akaike information criterion corrected (AICC) and Bayesian information Criterion $(B I C)$ for the real data set [2]. The best distribution corresponds to lower $A I C, A I C C$, and BIC values [7] and [13]:

$$
\begin{aligned}
& A I C=-2 \log L+2 k \\
& A I C C=A I C+\frac{2 k(k+1)}{n-k-1} \\
& B I C=-2 \log L+k \log n
\end{aligned}
$$

Where $\mathrm{k}$ is the number of parameters in the statistical model, $n$ the sample size and $L$ is the maximized value of the likelihood function for the estimated model.

\section{Data Analysis and Results}

The dataset used in this study consists of a sample of (118) lung cancer patients obtained from Salman and Farhan [6] and given in Table 2.

We can find the estimated value of the parameters and its confidence intervals for the distributions by using maximum likelihood estimation method as follows:

Table1: Maximum likelihood estimates parameters of the distributions

\begin{tabular}{|c|c|c|}
\hline Model & Estimates & $95 \%$ C.I \\
\hline Weibull & $\alpha=3.05$ & {$[2.62-3.54]$} \\
& $\lambda=373.58$ & {$[351.30-397.28]$} \\
\hline \multirow{3}{*}{ Gumbel } & $\mu=394.02$ & {$[374.42-413.61]$} \\
& $\beta=102.78$ & {$[89.521-118.01]$} \\
Exponential & $\lambda=337.15$ & {$[281.49-403.82]$} \\
\hline Log- logistic & $\beta=5.80$ & {$[5.72-5.88]$} \\
& $\alpha=0.26$ & {$[0.22-0.30]$} \\
\hline
\end{tabular}

The survival function estimations of time are obtained by substituting these estimated values of the parameters in equations (14), (20), (26), and (32) as shown in table 2. And the survival plots of the W.D, G.D, E.D and L.L.D are shown in figures 1, 2, 3, and 4 respectively. 
Ibn Al-Haitham Jour. for Pure \& Appl. Sci. 34 (2) 2021

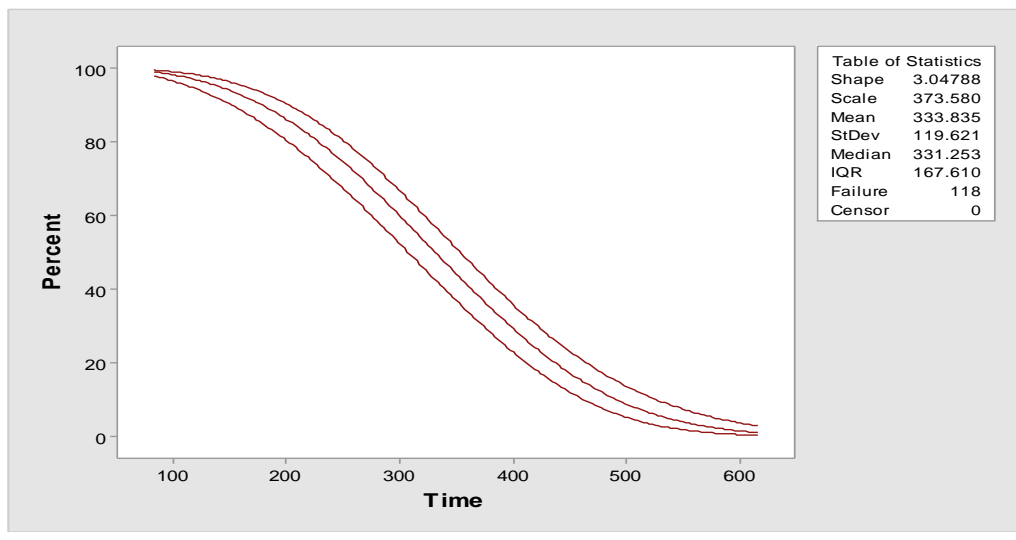

Figure 1: The curve of Weibull distribution for survival function

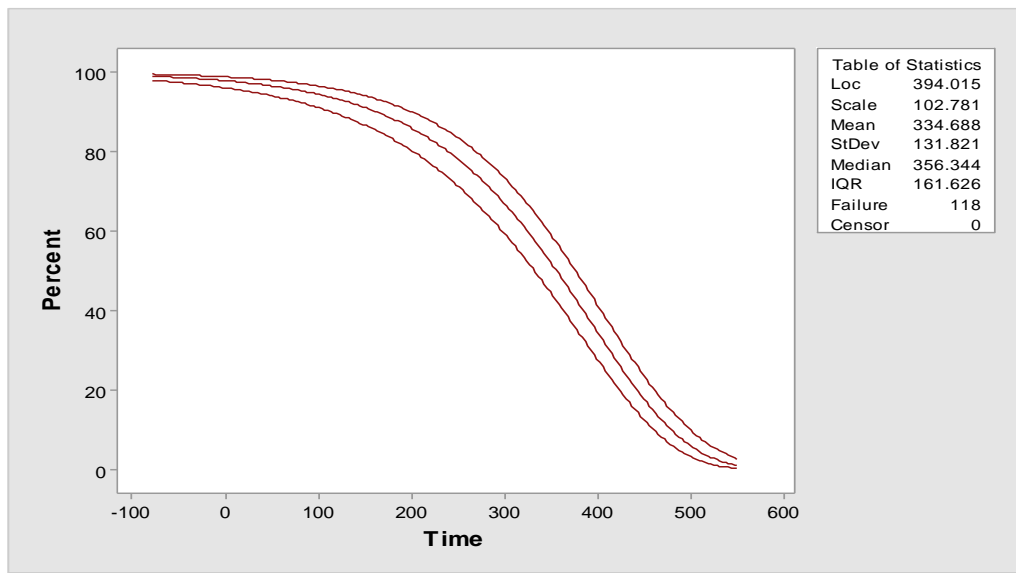

Figure 2: The curve of Gumbel distribution for survival function

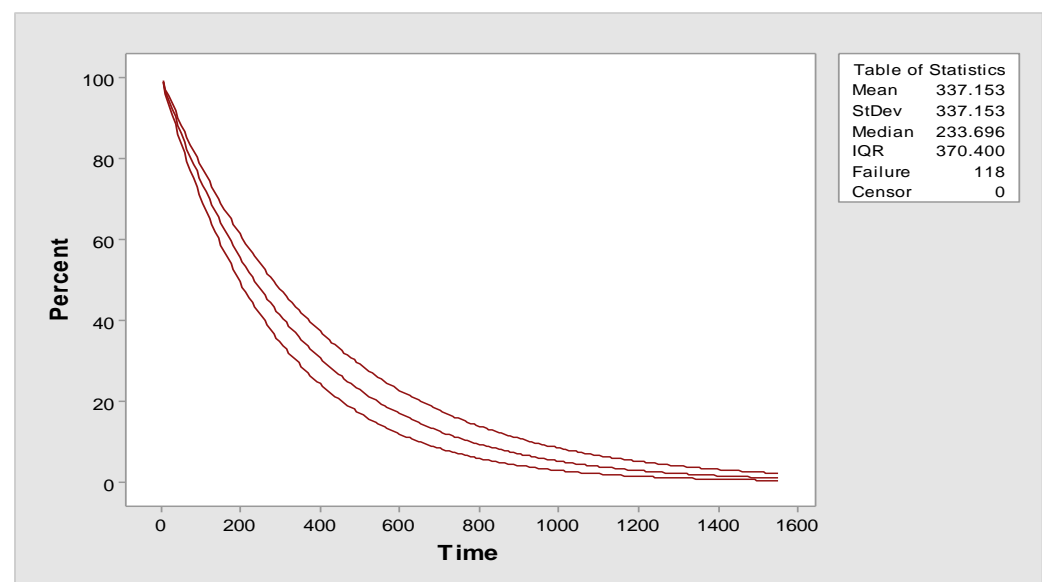

Figure 3: The curve of exponential distribution for survival function 
Ibn Al-Haitham Jour. for Pure \& Appl. Sci. 34 (2) 2021

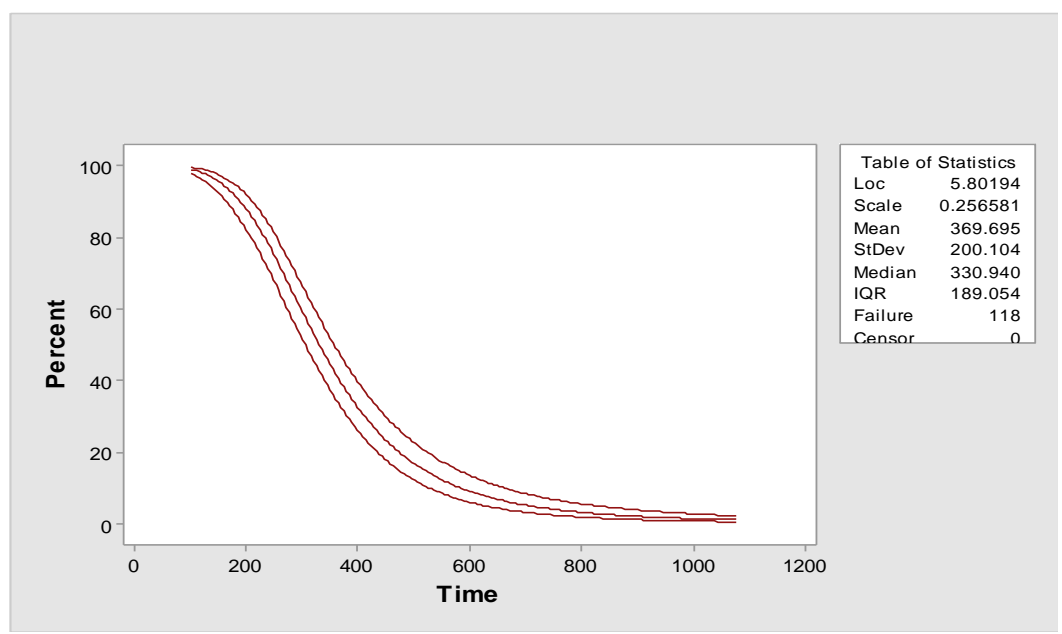

Figure 4: The curve of log logistic distribution for survival function

Table 2: Estimated values of the survival function [6]

\begin{tabular}{|c|c|c|c|c|c|c|c|c|c|c|c|}
\hline No. & $\begin{array}{l}\text { Time } \\
\text { /Day }\end{array}$ & $\widehat{\boldsymbol{S}}(W . D)$ & $\widehat{\boldsymbol{S}}(\boldsymbol{G} . \boldsymbol{D})$ & $\widehat{\boldsymbol{S}}(\boldsymbol{E} . D)$ & $\widehat{\boldsymbol{S}}(\boldsymbol{L} . D)$ & No. & $\begin{array}{l}\text { Time } \\
\text { /Day }\end{array}$ & $\widehat{\boldsymbol{S}}(\boldsymbol{W} . D)$ & $\widehat{\boldsymbol{S}}(\boldsymbol{G} . D)$ & $\widehat{\boldsymbol{S}}(\boldsymbol{E} . \boldsymbol{D})$ & $\widehat{\boldsymbol{S}}(\boldsymbol{L} . \mathrm{D})$ \\
\hline 1 & 3 & 1.000 & 0.978 & 0.991 & 1.000 & 60 & 341 & 0.469 & 0.550 & 0.364 & 0.471 \\
\hline 2 & 37 & 0.999 & 0.969 & 0.896 & 0.999 & 61 & 342 & 0.466 & 0.547 & 0.363 & 0.468 \\
\hline 3 & 72 & 0.993 & 0.957 & 0.808 & 0.997 & 62 & 345 & 0.456 & 0.538 & 0.359 & 0.460 \\
\hline 4 & 75 & 0.993 & 0.956 & 0.801 & 0.997 & 63 & 349 & 0.444 & 0.524 & 0.355 & 0.448 \\
\hline 5 & 91 & 0.987 & 0.949 & 0.763 & 0.994 & 64 & 354 & 0.428 & 0.508 & 0.349 & 0.435 \\
\hline 6 & 100 & 0.982 & 0.944 & 0.743 & 0.991 & 65 & 357 & 0.419 & 0.498 & 0.347 & 0.427 \\
\hline 7 & 103 & 0.981 & 0.943 & 0.737 & 0.989 & 66 & 363 & 0.400 & 0.477 & 0.341 & 0.411 \\
\hline 8 & 121 & 0.968 & 0.932 & 0.699 & 0.981 & 67 & 364 & 0.397 & 0.474 & 0.339 & 0.408 \\
\hline 9 & 127 & 0.964 & 0.928 & 0.686 & 0.977 & 68 & 364 & 0.397 & 0.474 & 0.339 & 0.408 \\
\hline 10 & 140 & 0.951 & 0.919 & 0.660 & 0.966 & 69 & 366 & 0.391 & 0.467 & 0.338 & 0.403 \\
\hline 11 & 154 & 0.935 & 0.908 & 0.633 & 0.952 & 70 & 367 & 0.388 & 0.464 & 0.337 & 0.401 \\
\hline 12 & 156 & 0.933 & 0.906 & 0.629 & 0.949 & 71 & 368 & 0.385 & 0.460 & 0.336 & 0.398 \\
\hline 13 & 164 & 0.922 & 0.899 & 0.615 & 0.939 & 72 & 371 & 0.376 & 0.449 & 0.333 & 0.391 \\
\hline 14 & 186 & 0.888 & 0.876 & 0.576 & 0.904 & 73 & 373 & 0.369 & 0.443 & 0.331 & 0.386 \\
\hline 15 & 211 & 0.839 & 0.845 & 0.535 & 0.853 & 74 & 380 & 0.349 & 0.419 & 0.324 & 0.369 \\
\hline 16 & 212 & 0.837 & 0.844 & 0.533 & 0.850 & 75 & 387 & 0.328 & 0.393 & 0.317 & 0.352 \\
\hline 17 & 213 & 0.835 & 0.842 & 0.532 & 0.848 & 76 & 387 & 0.328 & 0.393 & 0.317 & 0.352 \\
\hline 18 & 217 & 0.826 & 0.836 & 0.525 & 0.838 & 77 & 392 & 0.314 & 0.375 & 0.313 & 0.341 \\
\hline 19 & 218 & 0.824 & 0.835 & 0.524 & 0.836 & 78 & 393 & 0.311 & 0.372 & 0.312 & 0.339 \\
\hline 20 & 221 & 0.817 & 0.831 & 0.519 & 0.828 & 79 & 397 & 0.300 & 0.357 & 0.308 & 0.330 \\
\hline 21 & 221 & 0.817 & 0.831 & 0.519 & 0.828 & 80 & 399 & 0.295 & 0.350 & 0.306 & 0.325 \\
\hline 22 & 233 & 0.789 & 0.812 & 0.501 & 0.797 & 81 & 400 & 0.292 & 0.346 & 0.305 & 0.323 \\
\hline
\end{tabular}


Ibn Al-Haitham Jour. for Pure \& Appl. Sci. 34 (2) 2021

\begin{tabular}{|c|c|c|c|c|c|c|c|c|c|c|c|}
\hline 23 & 240 & 0.772 & 0.789 & 0.491 & 0.778 & 82 & 400 & 0.292 & 0.346 & 0.305 & 0.323 \\
\hline 24 & 241 & 0.769 & 0.798 & 0.489 & 0.775 & 83 & 401 & 0.289 & 0.343 & 0.304 & 0.321 \\
\hline 25 & 243 & 0.764 & 0.794 & 0.486 & 0.769 & 84 & 402 & 0.286 & 0.339 & 0.304 & 0.319 \\
\hline 26 & 249 & 0.748 & 0.784 & 0.478 & 0.752 & 85 & 407 & 0.273 & 0.322 & 0.299 & 0.309 \\
\hline 27 & 254 & 0.735 & 0.774 & 0.471 & 0.737 & 86 & 409 & 0.268 & 0.314 & 0.297 & 0.305 \\
\hline 28 & 266 & 0.701 & 0.749 & 0.454 & 0.701 & 87 & 419 & 0.242 & 0.279 & 0.289 & 0.285 \\
\hline 29 & 273 & 0.681 & 0.735 & 0.445 & 0.679 & 88 & 421 & 0.237 & 0.272 & 0.287 & 0.281 \\
\hline 30 & 276 & 0.672 & 0.728 & 0.441 & 0.669 & 89 & 421 & 0.237 & 0.272 & 0.287 & 0.281 \\
\hline 31 & 277 & 0.669 & 0.726 & 0.440 & 0.667 & 90 & 422 & 0.235 & 0.269 & 0.286 & 0.279 \\
\hline 32 & 278 & 0.666 & 0.724 & 0.438 & 0.664 & 91 & 422 & 0.235 & 0.269 & 0.286 & 0.279 \\
\hline 33 & 281 & 0.657 & 0.717 & 0.435 & 0.654 & 92 & 423 & 0.232 & 0.266 & 0.285 & 0.278 \\
\hline 34 & 290 & 0.630 & 0.695 & 0.423 & 0.626 & 93 & 427 & 0.222 & 0.252 & 0.282 & 0.270 \\
\hline 35 & 301 & 0.596 & 0.667 & 0.410 & 0.591 & 94 & 428 & 0.220 & 0.247 & 0.281 & 0.269 \\
\hline 36 & 301 & 0.596 & 0.667 & 0.410 & 0.591 & 95 & 430 & 0.215 & 0.242 & 0.279 & 0.265 \\
\hline 37 & 301 & 0.596 & 0.667 & 0.410 & 0.591 & 96 & 446 & 0.179 & 0.191 & 0.266 & 0.238 \\
\hline 38 & 302 & 0.593 & 0.665 & 0.408 & 0.588 & 97 & 450 & 0.171 & 0.178 & 0.263 & 0.232 \\
\hline 39 & 304 & 0.587 & 0.659 & 0.406 & 0.582 & 98 & 454 & 0.163 & 0.167 & 0.260 & 0.226 \\
\hline 40 & 304 & 0.587 & 0.659 & 0.406 & 0.582 & 99 & 416 & 0.249 & 0.289 & 0.291 & 0.291 \\
\hline 41 & 306 & 0.581 & 0.654 & 0.404 & 0.576 & 100 & 463 & 0.146 & 0.141 & 0.253 & 0.213 \\
\hline 42 & 307 & 0.577 & 0.651 & 0.402 & 0.573 & 101 & 470 & 0.133 & 0.123 & 0.248 & 0.203 \\
\hline 43 & 307 & 0.577 & 0.651 & 0.402 & 0.573 & 102 & 477 & 0.122 & 0.106 & 0.243 & 0.194 \\
\hline 44 & 308 & 0.574 & 0.649 & 0.401 & 0.569 & 103 & 481 & 0.115 & 0.097 & 0.240 & 0.189 \\
\hline 45 & 313 & 0.558 & 0.635 & 0.395 & 0.554 & 104 & 481 & 0.115 & 0.097 & 0.240 & 0.189 \\
\hline 46 & 313 & 0.558 & 0.635 & 0.395 & 0.554 & 105 & 483 & 0.112 & 0.093 & 0.239 & 0.186 \\
\hline 47 & 314 & 0.555 & 0.632 & 0.394 & 0.551 & 106 & 483 & 0.112 & 0.093 & 0.239 & 0.186 \\
\hline 48 & 318 & 0.542 & 0.621 & 0.389 & 0.539 & 107 & 497 & 0.092 & 0.066 & 0.229 & 0.170 \\
\hline 49 & 330 & 0.504 & 0.585 & 0.376 & 0.503 & 108 & 511 & 0.074 & 0.044 & 0.220 & 0.155 \\
\hline 50 & 331 & 0.501 & 0.582 & 0.375 & 0.499 & 109 & 512 & 0.073 & 0.043 & 0.219 & 0.154 \\
\hline 51 & 332 & 0.498 & 0.579 & 0.374 & 0.497 & 110 & 512 & 0.073 & 0.043 & 0.219 & 0.154 \\
\hline 52 & 332 & 0.498 & 0.579 & 0.374 & 0.497 & 111 & 516 & 0.069 & 0.038 & 0.216 & 0.150 \\
\hline 53 & 334 & 0.491 & 0.573 & 0.371 & 0.491 & 112 & 517 & 0.068 & 0.037 & 0.216 & 0.149 \\
\hline 54 & 334 & 0.491 & 0.573 & 0.371 & 0.491 & 113 & 519 & 0.066 & 0.034 & 0.215 & 0.148 \\
\hline 55 & 335 & 0.488 & 0.569 & 0.370 & 0.488 & 114 & 533 & 0.052 & 0.021 & 0.206 & 0.135 \\
\hline 56 & 335 & 0.488 & 0.569 & 0.370 & 0.488 & 115 & 534 & 0.051 & 0.021 & 0.205 & 0.134 \\
\hline 57 & 335 & 0.488 & 0.569 & 0.370 & 0.488 & 116 & 535 & 0.050 & 0.19 & 0.205 & 0.133 \\
\hline 58 & 335 & 0.488 & 0.569 & 0.370 & 0.488 & 117 & 540 & 0.046 & 0.016 & 0.202 & 0.129 \\
\hline 59 & 338 & 0.479 & 0.560 & 0.367 & 0.479 & 118 & 550 & 0.039 & 0.010 & 0.196 & 0.121 \\
\hline
\end{tabular}


Ibn Al-Haitham Jour. for Pure \& Appl. Sci. 34 (2) 2021

Table 3. Criteria for comparison

\begin{tabular}{|c|c|c|c|c|}
\hline Model & $-L L$ & AIC & AICC & BIC \\
\hline Weibull & 737.89 & 1479.78 & 1479.88 & 1479.92 \\
\hline Gumbel & 729.93 & 1463.86 & 1463.96 & 1464.00 \\
\hline Exponential & 804.82 & 1611.65 & 1611.68 & 1611.71 \\
\hline Log-logistic & 757.03 & 1518.06 & 1518.16 & 1518.20 \\
& & & & \\
\hline
\end{tabular}

The results in Table 3 indicate that the Gumbel distribution has the lowest AIC, AICC and $B I C$ values than the Weibull, log- logistic, and exponential. Hence Gumbel distribution leads to a better fit than the other three distributions.

\section{Conclusions}

From the practical work, it is concluded that the Gumbel distribution has the lowest $A I C$, $A I C C$ and BIC values than the Weibull, exponential, and log- logistic distributions. We conclude that the survival function for the lung cancer by using Gumbel distribution model is the best. And the expected values of the survival function of all estimation methods which are proposed in this article has been decreasing progressively with increasing failure times for lung cancer patients: this means that there is an opposite relationship failure times and survival function.

\section{References}

1. Mohammed, A. M., Shao T., Expected the Life Time for Heart Patients by Using Cox Regression Model, International Journal of Applied Research, 2015, 1, 9, 883-889.

2. Merovci, F., Transmuted Generalized Rayleigh Distribution, Journal of Statistics Applications and Probability, 2014, 3, 1, 9-20, htt://dx.doi.org/10.12785/jsap/030102.

3. Zhu, H. P. et al., Application of Weibull Model for Survival of Patients with Gastric Cancer, BMC Gastro enter bogy, 2011, 11,. 1, http://www.biomdcentral.

4. Abernethy, R. B., The New Weibull Hand Book, Ch.1, an over View of Weibull analysis, 2006, http://www.barringer1.com/pdf/Chpt1-5th-edition.pdf

5. Akhtar, M. ; Khan, A., A. Log- Logistic Distribution as a Reliability Model: A Bayesian Analysis, American Journal of Mathematics and Statistics, 2014, 4, 3, 162 170, doi:10.5923/j.ajms20140403.05.

6. Salman, A. N. ; Farhan, I. H., Estimate Complete the Survival Function for Real Data of Lung Cancer Patients, Ibn Al-Haitham Journal for Pure \&Applied Sciences, 2014, 27, 3, 531-541.

7. Yong, T., Extended Weibull Distributions in Reliability Engineering, PhD. Theses, 2004, National University of Singapore. 
8. Rausand, M. ; Hoyland, A., System Reliability Theory models, Statistical Methods, and Applications, $2^{\text {nd }}$ Edition, 2004, John Wiley \&Sons, INC, Canada, ISBN 0-47147133-X.

9. Chen, M.; Zhang, Z.; Cui, C., On the Bias of the Maximum Likelihood Estimators of Parameters of the Weibull Distribution, Mathematical and Computational Application, 2017, 22, 19, 1-18, 2017, doi: 103390/mca2210019.

10. Varela, J. Gorgoso ; Rojo-Alboreca, A., Use of Gumbel and Weibull Functions to Model Extreme Values of Diameter Distribution in Forest Stands, Annals of Forest Science, 2014, 71, 741-750.

11. Deka, D., Bhanita Das, B. ; Baruah, B. K., Transmuted Exponential Gumbel Distribution and its Application to Water Quality DATA, pak.j.stat.oper.res, 2017, 13, $1,115-126$.

12. Santana, T. V.; Ortega, E. M. M. Cordeiro, G. M ; Silva, G. O., The KumaraswamyLog- Logistic Distribution, Journal of Statistical Theory and Applications, 2012, 11, 3, 265-291.

13. Vllah, E. ; Shahazad, M. N., Transmutation of the Two Parameters Rayleigh Distribution, International Journal of Advanced Statistics and Probability, 2016, 4, 2 , 95-101, doi:10.1419/ijasp.v4i2.6100 